\title{
Humanismo y decisión estratégica, en el contexto de la transformación de Chile
}

doi: 10.33264/rpa.202101-09

David Mejías Pastene

Escuela de Administración UNIACC Facultad de Administración UNIACC

\section{Resumen}

El presente artículo pretende revalorar la visión holista, de conjunto, expresada en la filosofía, epistemología, ciencia, historia y el conocimiento teórico en general, para la eficacia de las decisiones estratégicas de las organizaciones y los países, en tiempos de transformaciones radicales como la que vive Chile en su proceso constituyente. En la historia generalmente los cambios primero se gestan en las vanguardias científico filosóficas, que poco a poco se van manifestando posteriormente en la cultura, el arte; después en los movimientos sociales, la contracultura que presiona para que estos postulados (no necesariamente revolucionarios, pero sí distintos al orden) se hagan carne en las instituciones. En este momento histórico está Chile, con la Administración como herramienta fundamental, que debe esgrimirse sin dogmas para poner toda la potencia de la inteligencia humana en la creación de modelos y esquemas administrativos para el logro del bien común y del desarrollo sustentable, en el contexto del respeto del otro como legítimo otro.

Palabras clave: administración, decisión estratégica, holismo, filosofía, fundamentos teóricos.

\begin{abstract}
These articles pretends reassess the holistic vision, overall, expressed in philosophy, epistemology, science, history and the theoretical knowledge in general, for the effectiveness of strategic decisions in organizations and countries, in times of radical transformations like the one who lives in Chile in its constituent process. In history generally changes first grow up in philosophical scientific vanguards that slowly they later manifested in culture, art; after in social movements, the counterculture that presses so that these postulates (not necessarily revolutionary, but distinct from order) are made meat in the institutions. In this historical moment is Chile, with the Administration like a fundamental tool that must be wielded without dogmas to put all the power of the human intelligence in the creation of administrative models and schemas for the achievement of the common good and sustainable development in the context of respect for the other as a legitimate other.
\end{abstract}

Keywords: Administration, Strategic decision, Holism, Philosophy, Theoretical fundaments. 


\section{Introducción}

Primero que todo hay que precisar que el objetivo de este artículo no es entregar avances importantes en el ámbito del pensamiento o de la epistemología, sino que más bien aplicar un esquema de explicación básico de causalidades que generan la realidad sociocultural, política, económica, etc., contemporánea, basadas fundamentalmente en las visiones dialécticas de Hegel y de la escuela alemana de Frankfurt, que hacen ver una especie de causalidad generadora de la realidad. La idea es darle un sentido práctico a la comprensión de esta cadena o proceso, en la fundamentación de una decisión estratégica que pueda tomar una organización pública o privada, un país, o una persona individual.

A grandes rasgos este proceso explicaría que la "emergencia" de las instituciones, de la realidad cultural actual, de los factores políticos y económicos, parte en las vanguardias científico filosóficas, de los espacios marginales de pensamiento libre, personificados en intelectuales, científicos, filósofos, estudiantes, académicos, artistas, que discuten o ponen en duda constantemente los conocimientos vigentes en distintas áreas, y de forma libre, sin ataduras de ninguna índole - aquí es aplicable el concepto de Enajenación necesaria de la marginalidad creadora que plantea Herbert Marcuse -, construyen sistemas simbólicos, de pensamiento, expresado en leyes, teorías, cosmovisiones o tendencias que poco a poco van ganando terreno primero en las manifestaciones artísticas, en la cultura, el arte alternativo o "contracultural" como se le denomina, bajando posteriormente a los movimientos sociales, a la sociedad en su conjunto, llegando a consolidarse ya definitivamente en las leyes, las instituciones, y la construcción de un nuevo orden, distinto al anterior.

Obviamente que la complejidad de la realidad sociocultural humana hace que no toda la realidad histórica empírica pueda ser explicada con este esquema tan simple y claro; y alguien podría decir que no necesariamente se da este proceso, porque pueden surgir factores de cambio directamente de la cultura popular, por ejemplo, del territorio, no necesariamente de la elite intelectual; o quizá la intelectualidad o "marginalidad creadora", como le llama Marcuse, se fije en una cultura preexistente como la Mapuche para redirigir el curso de la historia. Como es menester indicar, la ciencia social, y disciplinas como la Prospectiva, son de una complejidad tal que siempre se debe estar atento a las excepciones a la regla, y no forzar los hechos para acomodarlos a la teoría o al "esquema de explicación”.

Sin embargo, se pueden citar aciertos en el ámbito de la predicción (tan importante para la definición de las estrategias), por ejemplo el del teórico Español Manuel Castells, que predice los movimientos sociales del 2011, entre ellos, "Los Indignados" de España, y la "Revolución Pingüina" chilena, a través del análisis de las tendencias sociales y de la influencia de las tecnologías de la información y redes sociales en la 
población mundial, vistas desde una perspectiva holista, no solamente técnica. (Castells. 2009)

Otro ejemplo notable de aplicación del conocimiento teórico, filosófico, histórico, etc., para lograr proyecciones al futuro es la "Teoría Matemática de la Historia" (1967) del historiador español Alexandre Deulofeu, que encontrando constantes matemáticas en el desarrollo de los imperios, y en general de los procesos históricos humanos, realizó una proyección hasta nuestros días, y en ella auguraba un protagonismo de América Latina en el concierto mundial, y que Chile y Argentina serán "Faros de la Humanidad", por la forma de participación ciudadana y democrática que están alcanzando (Cuellilargo. 2019); prueba de ello es la instalación de la Asamblea Constitucional y la elección de una mujer mapuche como presidenta de la instancia constituyente; que más allá de ciertos problemas y controversias propios del contexto de agitación que genera la trascendencia del momento histórico; es un avance muy importante en el ámbito de la participación ciudadana y la inclusión de distintos sectores en las decisiones estructurales del país.

\section{¿Qué se entiende por humanismo?}

Según la Real Academia de la Lengua Española, Humanismo en su primera acepción es "cultivo o conocimiento de las letras humanas", lo que involucra su holismo, es decir su multi e interdisciplinariedad, en el sentido que las letras transportan o encarnan todo el conocimiento humano, de forma integral. Esto se confirma con la segunda acepción: "movimiento renacentista que propugna el retorno a la cultura grecolatina como medio de restaurar los valores humanos” (Mejías. 2019. Pág.2)

Aquí es importante poner de relieve la gran influencia que ha ejercido el paradigma positivista, que hace que lo que importe sean los hechos concretos, y que la mayor parte de las veces se muestran desagregados del todo, presentando una clara oposición al conocimiento teórico, sintético y holista, que poco a poco fue perdiendo crédito en la forma de ver el mundo que era la modernidad, recordando que rápidamente estamos pasamos a otra era, "postmodernidad", "sociedad del conocimiento", " 2 renacimiento", y muchas otras denominaciones que se le ha dado a la época contemporánea.

Las disciplinas más características del humanismo por su amplitud son la filosofía, la epistemología y la gnoseología. El conocimiento que busca el filósofo, por ejemplo, es holista, amplio, de la mayor cantidad de ramas del saber y disciplinas o aficiones, se busca la mirada sintética y abarcadora de muchas acciones superficiales.

La epistemología y la gnoseología, por su parte, son ramas de la filosofía; la primera se pregunta por la definición, fundamentos, paradigmas y métodos de las disciplinas 
científicas como la Administración y la Educación; y la gnoseología relaciona y sintetiza a los otros saberes, la relación con la historia, el arte, la ciencia, la tecnología, lo religioso, lo místico, lo cotidiano, lo laboral, etc.; en el fondo es una constatación de que el conocimiento es uno solo, de que todo está interconectado. ("Uno es todo", como decía Heráclito "el obscuro" - s. VI a.c.-)

Por consiguiente, representa la función contraria al positivismo que desagrega y especializa. La tendencia de la explicación teórica, humanista, es a ser sintetizadora, a formar en la cognición del alumno o aprendiz lo que la Teoría del Aprendizaje contemporáneo llama "Mapas Mentales", en los que pueda relacionar los nuevos conocimientos entregados por la academia o la vida, de forma crítica a partir de su propia visión de mundo, constituyéndose en un "sujeto crítico" y transformador de su yo interno y del entorno.

Este conocimiento holista es el que entrega el humanismo, recordando que es un concepto clave para el Proyecto Educativo UNIACC. La idea es que, entendiendo estas líneas abstractas e integradoras de la filosofía, la epistemología, la ciencia, la historia; podemos tener pistas claras para poder hacer proyecciones al futuro, que nos permitan tomar decisiones más eficaces en una organización sea pública, privada o de cualquier índole.

\section{Principales tópicos de la "vanguardia científico - filosófica"}

Para orientarse un poco en la línea argumentativa del texto, lo que se terminó de hacer fue explicar el proceso de transformación sociocultural que en el fondo es un patrón; como muchos otros que se dan en la naturaleza. La base central de esta visión es el filósofo alemán Georg Hegel (1770 - 1831), que plantea que la naturaleza de la racionalidad humana es la oposición de contrarios, la oposición de ideas.

Dentro de esta dialéctica idealista se genera la dinámica del espíritu universal que hace progresar la cultura. Si hay un freno a la marginalidad creadora se estanca el progreso del espíritu universal, que es más o menos lo que hace la Cultura de Masas cuando obliga al artista y al intelectual a someterse a las reglas del mercado - ya no hay mecenas y muy poco estado -, no le queda otra opción que transformar su obra en mercancía, y se genera la "sociedad unidimensional". Este orden está en crisis en la actualidad, y el germen de esa transformación son las vanguardias científico filosóficas.

Esa es la constante, y como en el Renacimiento del siglo XIV- XV, su contenido o médula era la naciente ciencia que con su evidencia empírica desmentía al dogma de fe católico; en la época contemporánea que algunos llaman " 2 renacimiento", su contenido es la "neociencia", la "neofilosofía”. Conocer estas tendencias es 
primordial, porque ayuda a comprender en profundidad los fundamentos de los movimientos sociales, los stakeholders, los target; la contracultura, lo que es vital para tomar decisiones en un contexto inmediato y en las proyecciones futuras, como la determinación de la Visión de las organizaciones privadas y públicas.

Enunciaré brevemente estas tendencias de la vanguardia científico - filosóficas, porque aquí lo que importa es su aplicación práctica en la decisión estratégica:

\section{a) Filosofía de Nietzsche y del existencialismo en general:}

Friedrich Nietzsche por muchos es considerado como el responsable del quiebre de la Modernidad, que según él la desplomó "a martillazos". Retoma la idea heraclitana del eterno flujo del cosmos, de la naturaleza. Nietzsche y el existencialismo dejan de entender al ser y la realidad como estáticos, y los entiende como un constante flujo, una constante transformación, devenir, que hace que las personas y organizaciones deben estar siempre adaptándose al cambio para sobrevivir. Los casos emblemáticos de Nokia, Blockbuster y Kodak, son ejemplos de empresas que no acomodaron sus estructuras administrativas y decisiones estratégicos al vertiginoso flujo de los cambios constantes del entorno, en este caso tecnológico. Por este motivo, las organizaciones deben adoptar una cultura del cambio donde además se fomente la disrupción, la innovación; que es la fuente de transformaciones que permiten la adaptación al entorno y la sobrevivencia de las organizaciones.

\section{b) Teoría de la Relatividad de Einstein:}

Básicamente la Teoría de la Relatividad, que es uno de los principales cuerpos teóricos que marcan el nuevo orden de cosas que se impone, significa la abolición de la verdad absoluta. No habría referentes únicos en el universo como planteaba Newton, básicamente porque el tiempo y el espacio son relativos a la velocidad que adquiera un objeto en el universo, que tiene su límite en la velocidad de la luz.

Este relativismo físico y cosmogónico se lleva a la cultura, con el "relativismo cultural". Toda cultura debe ser mirada en coherencia a su propia cosmovisión interna, no en relación a un supuesto paradigma cultural hegemónico como el europeo. Todo esto es muy coherente por ejemplo, con las palabras de Elisa Loncon Antileo, presidenta electa de la Convención Constituyente chilena, que señaló que el país avanza hacia ser un Estado plurinacional, plurilingüe, intercultural, y donde se respete lo territorial, el medio ambiente, las minorías; esto es una expresión clara de la plasmación de los valores de la neociencia, y del reconocimiento de las culturas ancestrales latinoamericanas, y sobre todo las andinas, entre ellas, la cultura mapuche. 


\section{c) Teoría de la Autopoiesis (Escuela Chilena):}

Esta escuela biológica surge en la década de los 70' y sus principales exponentes son Humberto Maturana y Francisco Varela. Expresan que la forma natural que tienen los seres humanos (su naturaleza) para interactuar unos con otros es el amor, que entienden como la "aceptación del otro como legítimo otro". Esto lo explican porque el amor (emoción que sólo se da en la especie humana), es lo que origina al lenguaje, que a su vez nos constituye como el tipo de ser humano que somos. Es decir "la aceptación del otro como legítimo otro" sería el fundamento y sentido de lo humano (Maturana. 1991).

Esta Autopoiesis biológica y la Teoría de la Relatividad en la física, en el fondo son bastante complementarias entre sí. El hecho que no haya un referente objetivo en el universo que de pie a una "verdad absoluta", para todos los tiempos y lugares, sino que más bien hay "realidades", "perspectivas" particulares dependiendo del lugar que tenga el observador en el cosmos. Esto trae como consecuencia para la Teoría de la Relatividad que todas las perspectivas y "verdades particulares" son legítimas. Por su parte la Autopoiesis que coloca a la "aceptación del otro como legítimo otro", interpreta con mucha claridad todos los movimientos a nivel mundial en contra del colonialismo y la visión etnocentrista europea, por ejemplo, que sometió a las culturas ancestrales y culturas distintas en general. En la actualidad, lo ancestral, como el ejemplo de la cultura mapuche en Chile, está imponiéndose como legítimo otro en el concierto de decisiones y diálogos estratégicos para construir el "nuevo orden", en el que tampoco se invalida la cosmovisión dominante, que sería una contradicción, sino que simplemente se coloca en la potencia necesaria para que haya un equilibrio de fuerzas que genere una convivencia más sana.

Todo esto se conecta también con el concepto de sustentabilidad del planeta que impulsa la Onu, y que definen instituciones internacionales independientes como ISO, que promueven la integración social, el respeto de los derechos humanos en las organizaciones, las condiciones laborales y el respeto por el medio ambiente, además del compromiso por el desarrollo de la comunidad donde se insertan las empresas u organizaciones en general.

Como ejemplo de lo anterior, y vinculándolo con la decisión estratégica en marketing se puede citar el caso del cambio de nombre de la marca "Negrita" a "Choquita", que causó mucha polémica porque dio a entender que el solo hecho de decirle negro a un negro podría interpretarse como una ofensa o denigración. Esto causó una serie de objeciones y burlas en redes sociales, y en el fondo evidenció un contrasentido en la intención de la empresa Nestlé de acomodarse a las nuevas tendencias de respeto e integración que exigen sobre todo los target mas jóvenes. Este es un buen ejemplo de cómo la incomprensión en profundidad de un fenómeno cultural o étnico puede 
llevar a tomar malas decisiones estratégicas. (Mejías. 2021)

\section{d) Unidad sujeto y objeto y superación de la "limitación cognitiva" humana:}

La antigua idea de Protágoras (siglo VI a. C.) de que "el hombre es la medida de todas las cosas", es postulada en la década de los 60" por la Escuela Alemana de la Gestalt y su "giro copernicano" en la teoría de la percepción, que señala que el ser humano tiene una "forma" de ver la realidad (comunicarse, aprender, interactuar, etc.) distinta a otras especies, y eso determina nuestra percepción del mundo.

Aquí se produce un quiebre muy importante, porque se deja de creer lo obvio: que las cosas (objetos, casas, mesas, etc.) se ven así porque son así; es decir, que tienen propiedades "objetivas" independiente del sujeto que las observa, como color, solidez, etc. Por el contrario, la Gestalt comienza a ver la relación con el entorno asumiendo que es el sujeto quien le imprime las propiedades a los objetos. Si vemos la mesa azul, no es porque el azul sea propiedad de la mesa, sino que nuestros ojos y nuestro cerebro le colocan ese color al objeto.

Aquí surge la pregunta: ¿De qué color es realmente la realidad?, que por ejemplo un gato vea la mesa gris, la mosca en distintos cubículos porque tiene muchos ojos; la verdad es que nadie con cuerpo puede saber cómo las cosas realmente son (color, densidad, geometría, etc.), porque tendríamos que ver sin ojos, por ejemplo, o tocar sin piel o manos; y esto es imposible. Sólo podemos ver y sentir lo que nos permite nuestro cuerpo; es decir, lo vemos a través de los sentidos y el cerebro nos construye una realidad que nos hace vivir en un mundo interpretado por él, y que es la base perceptual común de la humanidad. (Todos los seres humanos tenemos el mismo cerebro, no hay ninguna diferencia por etnias).

Aquí el concepto de objetividad se desdibuja; y desde este momento primará la subjetividad; la postura de Protágoras - presocrático - por ejemplo, contrario a la metafísica griega clásica - Sócrates, Platón y Aristóteles -, que decía "el hombre es la medida de todas las cosas"; Estos principios que la Gestalt desprende del estudio del cerebro humano, llevados a la comprensión sociocultural significa que cada persona construye el mundo y la realidad en función de su propia estructura cognitiva determinada a su vez por su particular historia personal, la influencia de los medios de comunicación, su herencia congénita, sus conocimientos previos, etc.

Esto indica en el fondo que a pesar que vivimos compartiendo los mismos objetos físicos, el mismo mundo físico; el significado que le damos a las cosas - la idea que construimos en la mente cuando nos enfrentamos a un objeto-, y por tanto el mundo que construimos (realidad subjetiva), dependerá de nuestra estructura cognitiva. 
Otra característica importante del cerebro humano, que se le podría denominar como una especie de "limitación” (entendiendo que el cerebro es una máquina biológica), es que para su funcionamiento es muy importante la válvula selectora, que deja entrar entre 5 a 9 datos al cerebro en la memoria inmediata o de "corto plazo", y con esos datos construimos la impresión de la realidad externa, o nuestra imagen.

Esta válvula selectora nos da la sensación de estabilidad, de orden de la realidad externa porque nos impide dejar entrar muchos datos a la vez; pero la verdad es que el entorno externo es caótico, multifactorial, ultra complejo. Si por algún motivo la válvula del cerebro deja entrar más información que lo normal- en una sala, por ejemplo: el número de baldosas del piso, que colores tiene cada una, cuántas lámparas había, qué otras personas habían ella, las manchas en la muralla, las pelusas de la polera de tal sujeto, etc., etc., etc., - nos podemos sobresaturar hasta la taquicardia incluso; porque en el fondo es andar por el caos real de la naturaleza sin ningún filtro o escafandra.

Cuando la Gestalt habla de "la forma", toma como referente al filósofo alemán Inmanuel Kant, quien en su magistral libro - según muchos el más importante de la filosofía mundial - "Crítica a la razón pura", señala que el hombre tiene una "forma” de percibir la realidad externa, y el primer filtro son las "sensaciones puras a priori", y aquí están comprometidas los sentidos de tiempo y espacio que tiene el cerebro, para darle un marco matemático y geométrico a la percepción de la realidad. Para que estas sensaciones puras a priori operen, no tenemos que hacer nada; son funciones reflejas del cerebro o del cuerpo.

En este sentido, la matemática y la geometría son asuntos internos del cerebro, la lógica, en general las ciencias formales; no tienen existencia en la realidad física, más bien tienen una naturaleza abstracta, inmaterial, que sólo habitan en nuestro cerebro. Esta sensibilidad pura kantiana es la que nos hace, o nos obliga mejor dicho a construir imágenes y esquemas de las cosas con una consistencia lógica, una cuadratura geométrica - matemática en los ejes de tiempo y espacio que es la forma particular de "leer" la realidad externa, como las moscas tienen otra muy distinta, por ejemplo. Todo este mecanismo nos condiciona mucho a tener la sensación de certeza cuando creemos llegar a la "madurez", y el mito de que "ahí terminamos de aprender"; y que no cambiamos más de opinión, porque eso es "malo", que es sinónimo de inconsistencia.

En síntesis, basándose en estos conocimientos neurocientíficos y de la estética de Kant, sería el propio cerebro humano el que nos lleva primero a ver orden en el caos; y segundo nos condiciona a darle solidez lógica y coordenadas dentro de un esquema espacio temporal - aritmética y geometría - interno (formas puras de la sensibilidad) a esas limitadas cosas que incorporamos a nuestra memoria, más bien por nuestras 
propias motivaciones o intereses internos.

Aquí viene lo medular: la más importante máxima de la ciencia cognitiva aplicada a la Administración y en general a toda actividad humana, es que hay que vencer la limitación del cerebro y saber que hay una posibilidad infinita de construcción de cosmovisiones a través del lenguaje, que dota al ser humano de una capacidad creativa infinita; y lo hace superar su determinación instintiva, construyendo la dimensión cultual, fuente del sentido de su existencia. (Chomsky, 1984). Estas distintas cosmovisiones que pueden tener tribus, pueblos, civilizaciones, etc., representan distintas dimensiones desde las cuales se interpreta la realidad, y con sus propias lógicas de articulación interna; estas perspectivas culturales tienden a ser inconmensurables entre sí.

Inconmensurable significa que cada elemento de una cultura, como el simbolismo de la Cultura Mapuche por ejemplo, no puede compararse bajo los mismos parámetros que la misma temática u elemento de la cultura occidental, chilena, por ejemplo; sino que cada elemento primero hay que comprenderlo en profundidad dentro del sistema propio de su cultura - su propia hermenéutica interna como diría Husserl, padre de la Fenomenología -, para después establecer un cruce, analogía o comparación con algún grado de validez. Si llevamos esto a la Convención Constitucional, por ejemplo; el cruce intercultural para aunar cosmovisiones y redactar una constitución responde mucho a los aspectos filosófico - científicos recién expuestos.

Para entender los fenómenos interculturales, es muy importante la antropología disciplina que es trascendental para toda carrera u actividad que trabaje con personas -, significa que cada sujeto que tenemos al frente es una ventana o puerta abierta a otra dimensión; una invitación a ponerse sus lentes con los que define el mundo, a estar consciente de su "otredad"; de esta forma, y en la medida que se comprenden otras dimensiones o posibilidades de interpretación de la realidad se va alcanzando la sabiduría, traducida en comprensión, respeto, porque se adquiere una visión más panorámica y multidimensional; un poquito más cercanos a la verdad global o absoluta, a la que no llegaremos nunca, por lo menos con el cuerpo que nos contiene.

De esta forma, toda la línea de la neociencia y la neofilosofía, que constituyen los mapas teóricos o lineamientos abstractos fundamentales, tiende a la superación del dogmatismo, que en el ámbito de la Administración, lleva a superar las limitaciones dogmáticas que impone el cerebro humano y el criterio de certeza. El neoliberalismo considera aberrante la participación del estado en el mercado; el comunismo ve en la empresa privada y hasta en las cooperativas amenazas a sus esquemas rígidos de administración; hasta el anarquismo es dogmático cuando no ve nada favorable y útil en el Estado. Lo mismo podemos ver en las fracciones políticas que no conciben que 
el posteo en redes sociales o el sufragio sean formas legítimas y efectivas de lograr transformaciones reales. Todos estos dogmatismos y fanatismos deben ser superados, sobre todo en el contexto de la instancia constituyente que vive el país, y que está generando tanta polémica. La pronta asimilación de estos principios; y un correcto acompañamiento al proceso de transformaciones podrían llevar a la Convención y al país a un muy buen puerto y quizá transformarse en ese "Faro" que profetizaba Deulofeu. (Matemática de la Historia).

\section{e) Filosofía del lenguaje:}

En este acompañamiento a los cambios podría ser muy apropiado el "Coaching Ontológico" del filósofo chileno Rafael Echeverría, que toma la Teoría de la Autopoiesis de Maturana y Varela para expresar que el "lenguaje genera ser", que el lenguaje humano no es una mera herramienta que nos sirve para describir pasivamente la realidad externa, sino que es un mecanismo poderoso que tiene el ser humano para transformar su realidad interna (ser) y la realidad externa (sociedad, mundo, entorno). En este sentido, muchas veces basta que declaremos algo para que se haga realidad. Esto nos conecta muy fuertemente con los lineamientos estratégicos, la visión de las organizaciones o de un país.

Las conversaciones que mantienen los colaboradores en las secciones de una empresa o los ciudadanos en las calles de una ciudad o pueblo, son las que nos proyectan a un futuro y crean realidad. "Queremos ser una organización que se encumbre en los ratings de Responsabilidad Social Empresarial en tal área", o llegar a estar en todos los escritorios del mundo, como se propuso Microsoft o Google; las declaraciones con respecto al futuro (visión), si se hacen con fuerza y convicción adquieren "propiedades mágicas" en el sentido que funcionan como transformadoras de la realidad, como si las palabras tuviesen propiedades alquímicas; esto revitaliza la vigencia del idealismo, porque muchas veces son las ideas las que genera realidad. Todo esto es el componente lingüístico de una institución, su alma; las entidades abstractas que rigen a los cuerpos físicos concretos, empíricos.

\section{Conclusión}

El propósito central de hacer este punteo de principales tendencias teóricas, científicas, filosóficas y culturales que se instalan en el macro entorno de las organizaciones, es constatar la fuerte correspondencia que hay entre la vanguardia científico - filosófica del mundo, y las tendencias artísticas, sociales, culturales que se van gestando en el planeta, y la presión que hace la "contracultura" para que estas tendencias se concreten en la institucionalidad y construyan un "nuevo orden". 
En este sentido, se evidencia que el proceso que vive el mundo y Chile en particular, es más bien la materialización institucional de las tendencias de la vanguardias científico filosóficas y de la contracultura. Por este motivo, estar pendiente de estas tendencias permite adelantarse al futuro, y tomar decisiones estratégicas que recojan estás líneas abstractas, y de esta forma no encuentren a las organizaciones y al país desprevenidos como ocurre generalmente en la historia, y se pueda adaptar correctamente la institucionalidad a los nuevos principios, respetando la diversidad, las visiones críticas, las minorías, evitando los conflictos, la confrontación y el dogmatismo, para dejar a la libertad creativa humana a sus anchas para diseñar los esquemas administrativos apropiados que favorezcan el bien común, en una sociedad de iguales en un ambiente de tolerancia y de respeto.

Volviendo a la Teoría de Matemática de la Historia de Alexandre Deulofeu, que aplica esta ciencia formal en la historia, encontrando patrones numéricos que le sirvieron para predecir la caída de la Unión Soviética, el ascenso de China y Alemania, la actual decadencia de EE.UU, y la supuesta "era de América Latina", y del cono sur: Chile, Argentina y Uruguay, principalmente como "faros de la humanidad", porque en ellos se vivirán procesos cada vez más crecientes de democratización, pluralismo y diversidad. La verdad es que esta Teoría Matemática puede ser cuestionable, pero si nos preguntamos, ¿qué tienen en común las culturas argentina y chilena?: la Cultura Mapuche, que con su cosmovisión de respeto al ecosistema, al considerarse parte indivisible de él, se conecta muy bien con los principios de la sustentabilidad y la gobernanza, que propicia la Onu, organizaciones privadas, la comunidad y el territorio en general para salvar al planeta de la eventual extinción de la especie por destrucción ecológica.

Aunque no sea completamente certificable, nada impide al país colocarse esa meta de ser un "faro de la humanidad" en un tiempo cercano, elaborando una nueva constitución en base a los principios de la tolerancia y del "respeto del otro como legítimo otro", que el maestro Humberto Maturana predicaba. Si en Chile se pudiese demostrar al mundo que a pesar de las dificultades y turbulencias propias de un proceso de cambios drásticos, se llegó a buen puerto con una carta fundamental representativa de todos los intereses de los distintos grupos y sectores; este logro sería un elemento exportable que quizá nos hiciera pasar a ser un referente. El acompañamiento al cambio y el coaching ontológico de la Escuela Chilena podrían ser un buen aliado para esto.

Finalmente, apuntando al entorno concreto de la Universidad UNIACC, su sello artístico, transformador y creativo es un caldo de cultivo ideal para canalizar las transformaciones que se están gestando; y la Escuela de Administración, una instancia propicia para imaginar nuevos espacios y formas de convivencia. En el 
ámbito de la Educación y la Administración, y en todas las áreas del saber, mientras en el ocaso del antiguo paradigma se pensaba que ya estaba todo escrito, en la irrupción de la nueva era está todo por escribir, por crear, rectificar y transformar.

\section{Referencias}

Cárdenas, M. (2015). Focos y trayectorias de la Comunicación Social. Ediciones Rutafuturo.

Castells, M. (2009). Comunicación y poder. Alianza Editorial.

Chomsky, N. (1984). Lingüística cartesiana. Editorial Gredos.

Cuellilargo. (2019, 30 de septiembre). La Matemática de la Historia [Video]. YouTube. https://www.youtube.com/watch?v=dyLNaurtTZ8

Mejías, D. (2012). Comunicación postmoderna: bases científicas y filosóficas. Ediciones Caballo de Mar.

Mejías, D. (2019). Reflexiones en torno al humanismo, pilar central del proyecto educativo UNIACC, Revista Pensamiento Académico, 2(1). 111-118. https://doi.org/10.33264/rpa.201901-09

Mejías, D. (2021, 3 de agosto). Por una negrita se volvieron locos. Blog Uniacc. https://blog.uniacc.cl/por-una-negrita-se-volvieron-locos 


\section{David Mejías Pastene}

Periodista y Licenciado en Ciencias de la Comunicación (Ucn); Magister en Educación Superior (Uniacc); egresado de Magister en Filosofía de las Ciencias (Usach). Autor del libro "Comunicación Postmoderna: Bases Científicas y Filosóficas” (2012), y de artículos en revistas académicas especializadas. Realiza frecuentemente cátedras de Comunicación Social, Comunicación Interpersonal, Comunicación Corporativa, Semiótica, Ética y Metodología de la Investigación.

Publicaciones e investigación:

- Proyecto de investigación "Perfil cultural del estudiante de Ingeniería Comercial, para lograr Aprendizaje Significativo", en año 2018, y del proyecto "Visualización gráfica hipertextual del Perfil Cultural de los/las estudiantes de Artes Visuales e Ingeniería Comercial para el Departamento de Investigación y Desarrollo de Uniacc.

- Libro “Comunicación Postmoderna: Bases Científicas y Filosóficas”, publicado por Ediciones Caballo de Mar, Lampa, Chile, año 2012, 207 págs.

- Investigador del proyecto "Comportamiento del mercado laboral, para carreras de comunicación y carreras técnicas de nivel superior"del I.P. Los Leones.

- Proyecto de investigación "Fundamentos de la Teoría de la Comunicación Postmoderna”, financiado por el I.P. Los Leones, cuyo mejoramiento es el texto "Comunicación Postmoderna”, de Ediciones Caballo de $\operatorname{Mar}$ (2012).

- Tesis "Teoría de la recepción activa: una propuesta de análisis metodológico en el nuevo enfoque comunicacional”, con la que se obtuvo el grado de Licenciado en Ciencias de la Comunicación en la Universidad Católica del Norte, calificado con la nota máxima. 